\title{
Concurrent Achilles tendon vibration and tibial nerve stimulation to estimate persistent inward current strength in motoneurons
}

\author{
Denis César Leite Vieira (1), Amilton Vieira (1), Matheus Avelino dos Santos (1), \\ Rafael Rodrigues da Cunha (1), Victor Lage (1), Anthony J. Blazevich (2), Martim \\ Bottaro (1) \\ (1) College of Physical Education, University of Brasilia, Federal District, Brazil; (2) Centre \\ for Exercise and Sports Science Research, School of Medical and Health Sciences, Edith Cowan \\ University, Joondalup, Australia.
}

This article is distributed under the terms of the Creative Commons Attribution Noncommercial License (CC BY-NC 4.0) which permits any noncommercial use, distribution, and reproduction in any medium, provided the original author(s) and source are credited.

\begin{abstract}
Vibratory (Tvib) and sustained (Tsust) torque responses to concurrent Achilles tendon vibration and neuromuscular electrical stimulation applied over the muscle belly (vib+stim) are used as indicators of motoneuron facilitation and, theoretically, persistent inward current strength. However, neuromuscular electrical stimulation (NMES) applied to the nerve trunk may potentiate motoneuronal excitability more than muscle belly NMES, yet it remains unclear whether NMES applied over the nerve evokes robust Tvib and Tsust responses when used during the vib+stim protocol. This study tested whether a nerve-targeted vib+stim protocol elicits Tvib and Tsust responses in the ankle plantar flexors with acceptable intra- and inter-session reliability. Fifteen men performed the vib+stim protocol with NMES applied over the tibial nerve three times across two sessions; twice in a single session (5-min apart) to test intrasession reliability and then again after $48 \mathrm{~h}$ to test intersession reliability. Intraclass correlation coefficients (ICC3,1), within-participant coefficients of variation (CV) and pairwise comparisons were used to verify relative and absolute reliability as well as systematic bias. Thirteen men presented Tvib and Tsust responses (response rate of 87\%). Intrasession Tvib and Tsust ICCs were $>0.73$ but inter-session ICCs were $<0.5$. Although no systematic bias was detected ( $>>0.05)$, both intra- and inter-session CVs were large $(>10 \%)$ for Tvib and Tsust. The Vib+stim protocol with NMES applied over the nerve evoked Tvib and Tsust in almost all participants, but presented a large intra- and inter-session variability. The method does not appear to be effective for assessing motoneuron facilitation in the plantar flexors.
\end{abstract}

Key Words: Motoneuron; neuromuscular electrical stimulation; intraclass correlation coefficient; coefficient of variation; neuromuscular system.

Eur J Transl Myol 31 (4): 10045, 2021 doi: 10.4081/ejtm.2021.10045

Facilitatory modulation at the motoneuron can be exerted by the development of persistent inward currents (PIC), ${ }^{1,2}$ which are depolarizing currents caused by voltage-sensitive $\mathrm{Na}^{+}$and $\mathrm{Ca}^{2+}$ channels largely residing in the motoneuron dendrites. ${ }^{3}$ These channels remain open while the motoneuron membrane potential remains above the threshold for PIC activation, ${ }^{2}$ causing a sustained motoneuron depolarization and allowing the motoneuron to fire at higher frequencies than would be achieved solely through ionotropic (non-PIC) activation. ${ }^{1,2}$ PICs also allow the motoneuron to remain firing when synaptic input from supraspinal and reflexive pathways decreases below the level at firing onset; i.e. PICs alter the motoneuronal input-output relationship. ${ }^{1,4}$
Given the influence of motoneuron firing on muscular force production, ${ }^{5,6}$ PICs are fundamental to achieving high force levels. ${ }^{7,8}$ They play an important role in some clinical conditions, including spasticity. ${ }^{2}$ The paired motor unit technique is the most recognized and accepted method for PIC estimation in vivo in humans. ${ }^{9}$ In this method, pairs of motor units of different firing thresholds are tracked during a ramped isometric contraction, ${ }^{4}$ and based on bistable firing behavior of motoneurons, PIC strength in a higher-threshold motor unit (i.e. a 'test' unit) is estimated as the difference in firing rate of a lowerthreshold ('control') motor unit at the points of recruitment and derecruitment of the higher-threshold motor unit. ${ }^{4,10}$ That is, PICs are considered to be greater when the higher-threshold units continue to fire in the 


\section{Concurrent Achilles tendon vibration and tibial nerve stimulation}

Eur J Transl Myol 31 (4): 10045, 2021 doi: 10.4081/ejtm.2021.10045

downward slope of the ramp contraction to lower firing rate levels (and usually lower force levels) of the control unit. However, the paired motor unit technique requires the use of either invasive intra-muscular electromyography (EMG) or high-density surface EMG systems with the associated use of complex motor unit decomposition algorithms. Furthermore, the technique requires the production of voluntary muscle contraction, which themselves influence PIC activation through the release of neuromodulators such as serotonin, ${ }^{11}$ and contractions must be accurately produced without activation of antagonist muscles that might inhibit PICs. ${ }^{12}$ Accurately producing ramped contractions may be especially difficult in many clinical patients. ${ }^{13}$ Due to these limitations, the paired motor unit technique may not always be feasible in some clinical or research settings.

An alternative method to estimate PICs in vivo in humans is required. One possibility that has yet to be fully validated is to assess the neuromuscular response to tendon vibration, ${ }^{1,2}$ whereby an increase in motoneuron firing frequency and force output not only during, but also after cessation of tendon vibration may be indicative of PIC activation. ${ }^{2,14}$ Whilst, isolated tendon vibration usually recruits only low-threshold motor units, which in turn may result in a small muscle force output, ${ }^{1,12}$ researchers have simultaneously imposed neuromuscular electrical stimulation (NMES) and tendon vibration to recruit higher-order motor units and thus produce greater forces. ${ }^{15}$ Subsequently, 2-s NMES bursts have been imposed over tendon vibration ("stim+vib" technique; 33 s vibration at 70 to $115 \mathrm{~Hz}$ ) and the torque developed during vibration after NMES cessation (vibratory torque; Tvib) as well as the sustained involuntary torque output after tendon vibration cessation (sustained torque; Tsust) taken as estimates of motoneuron output facilitation. ${ }^{1,14}$ Although several physiological mechanisms may influence the facilitation, it has been considered to be strongly influenced by PIC activation because it displays many hallmarks of PIC behavior, including joint angle (i.e. muscle length) dependence, warm-up (increasing effect as stimulus continues), sustained involuntary muscle activity (EMG) and force production in the absence of synaptic input (i.e. self-sustained motor unit firing), and inhibition by antagonist muscle activation (e.g. Trajano et al. $)^{1}$ However, direct proof of the input of PICs to the test outcomes has not yet been obtained. Although Tvib and Tsust have been recognized as markers of motoneuron facilitation, and possibly PIC activation, ${ }^{1,14}$ further research is required to determine their reliability as a potential test of PIC strength (or facilitation more broadly) in human motoneurons as well as the potential clinical role for assessing PICs in aging, rehabilitation and patient populations. Moreover, some methodological procedures still need to be clarified. For instance, the reliability of Tvib and Tsust have only been reported from data captured in the same session (i.e. intra-session reliability; Trajano et al.). ${ }^{1}$

However, as Tvib and Tsust measurements are required between days to assess both acute and chronic effects of disease, disuse, exercise or nutritional interventions, inter-session reliability needs to be ascertained. Furthermore, Trajano et al. ${ }^{1}$ and Kirk et al. ${ }^{14}$ applied NMES to the muscle belly, which may be less efficient than NMES over the nerve trunk to recruit motor units through central pathways. ${ }^{16}$ Indeed, afferent Ia fiber traffic to the motoneurons may also be higher during NMES applied over the nerve than the muscle belly, at least when low forces are evoked by the NMES. ${ }^{16,17}$ Furthermore, nerve stimulation may recruit motor units more broadly within a muscle, rather than only those that lie superficially, closer to the stimulating electrodes. Thus, the vib+stim protocol might theoretically be more potent when NMES is applied over the nerve than the muscle belly. However, it remains unclear if the vib+stim protocol using nerve stimulation would evoke Tvib and Tsust responses as well, and as reliably, as with muscle belly NMES.

Therefore, the primary aim of this study was to determine whether the vib+stim protocol performed with NMES applied to the tibial nerve could elicit significant Tvib and Tsust responses from the plantar flexor muscles. A second aim was to determine both the intra- and intersession reliability of the vib+stim protocol when using tibial nerve stimulation. As motor units exhibit differences in bistable behavior, with fully bistable units showing prolonged firing behavior after activation (probably lower-threshold, fatigue resistant units) and partially bistable units ceasing relatively rapidly (<3s; Lee and Heckman), ${ }^{18}$ Tvib and Tsust responses were assessed at multiple time points up to $4 \mathrm{~s}$ after cessation of NMES and up to $3 \mathrm{~s}$ after cessation of tendon vibration to determine whether reliability is affected by the measurement time point.

\section{Materials and Methods}

\section{Participants}

Fifteen young, physically active men without known neuromuscular, metabolic, or cardiovascular impairments completed one familiarization and two experimental sessions. However, a notable warm-up effect (i.e. a difference in torque during vibration after the first NMES burst vs. the fifth/last NMES burst [Tvib]) or a sustained torque response was clearly detected in at least one trial in only 13 participants. Therefore, 13 "responsive" men were subsequently included in the reliability analysis $(25.2 \pm 5.5 \mathrm{y} ; 76.6 \pm 9.0 \mathrm{~kg} ; 1.73 \pm 0.1$ $\mathrm{m} ; 25.6 \pm 3.2 \mathrm{~kg} / \mathrm{m}^{2}$ ). These participants were instructed to avoid vigorous physical activity and caffeine $48 \mathrm{~h}$ prior the experimental sessions, and to not take medications or food supplements throughout the study period. All procedures were approved by the Institutional Review Board (12630519.6.0000.5650) and were performed in accordance with the Declaration of Helsinki. The informed consent was obtained from all participants prior to participation. 


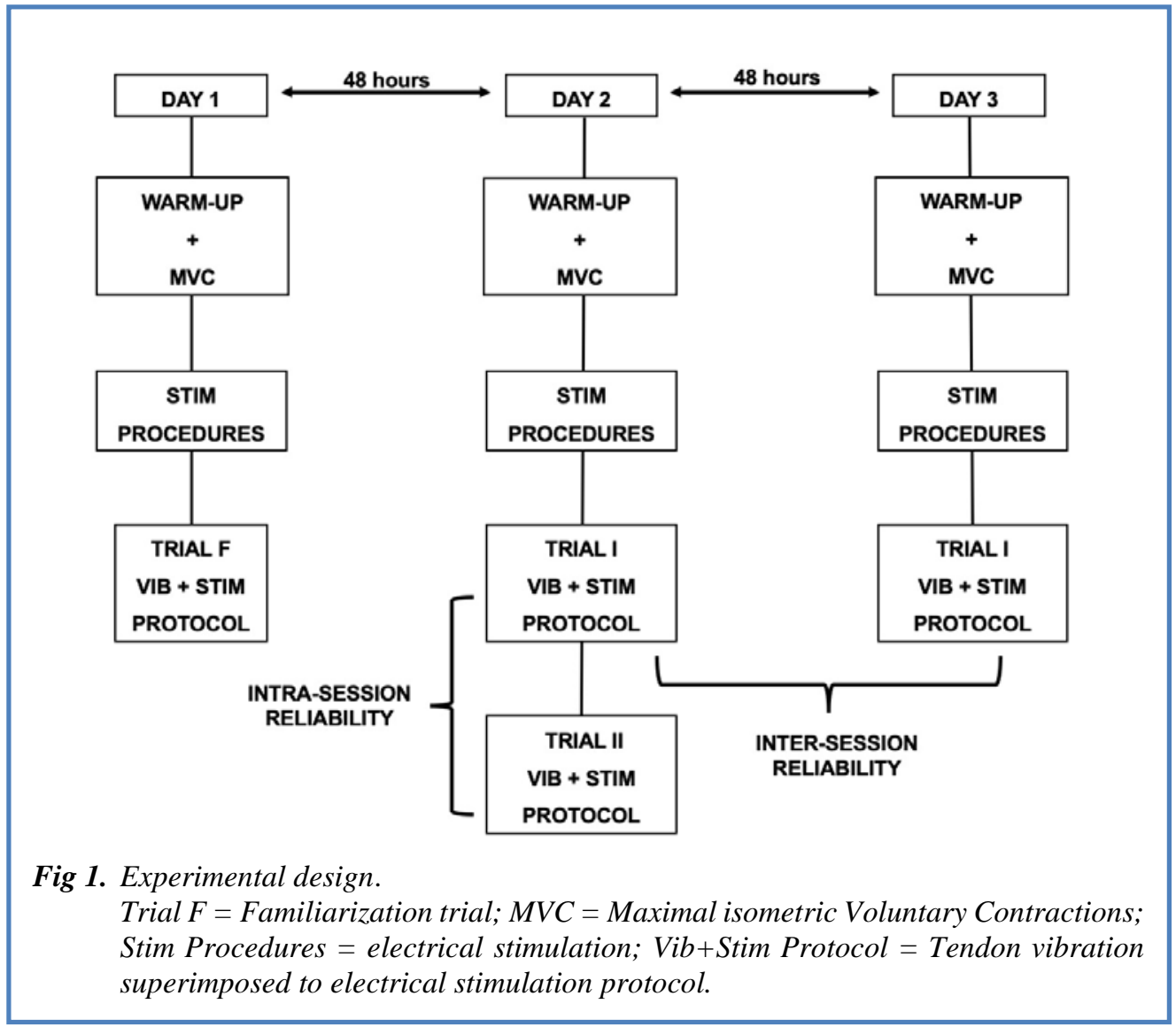

\section{Experimental design}

The experimental design is shown in Figure 1. Participants visited the laboratory on three occasions. On Day 1 the participants were familiarized with all experimental procedures. On Day 2 (Experimental Session 1), the participants performed the vib+stim protocol twice with a 5-min passive recovery in order to determine the intrasession reliabilities of Tvib and Tsus. After $48 \mathrm{~h}$, they performed the vib+stim protocol again to allow assessment of intersession reliability (Experimental Session 2).

\section{Experimental procedures}

All procedures were performed on the ankle joint of the right leg. The participants were seated in isokinetic device with the knee fully extended and ankle dorsiflexed to $10^{\circ}$ since vib+stim protocol demonstrates a joint-angle dependence 1. Initially, participants sat in the chair of an isokinetic dynamometer (Biodex System 4 Pro, Biodex Medical System, Shirley, NY) with the right knee extended, hips flexed to $85^{\circ}$, and ankle joint in $10^{\circ}$ dorsiflexion. After seat belts were applied across the chest to minimize extraneous movement, participants performed a warm-up of five isometric plantar flexions at $20,40,60,80$, and $100 \%$ of perceived maximal effort before performing three maximal voluntary isometric plantar flexor contractions (MVC) with 1 min of passive rest. Subsequently, a constant-current electrical stimulator (Nicolet Viking Quest, Natus Medical Incorporated, Pleasanton, CA) was used to deliver an electrical square-wave stimulus (1-ms pulse width) to the tibial nerve. The cathode was placed on the popliteal fossa at a point that evoked the greatest motor response and the anode was placed over the patella. The intensity necessary to induce an isometric contraction at $20 \%$ of MVC (measured during familiarization) with a 2-s $20-\mathrm{Hz}$ tetanic train was set daily but used in all stimulations performed within a given session; this $20 \%$ MVC torque level was used in previous studies using muscle belly NMES. ${ }^{1,14}$ The participants then completed the stim+vib protocols.

Tendon vibration superimposed with NMES electrical stimulation (stim+vib)

Tsus and Tvib were measured during the stim+vib protocol. The Achilles tendon was mechanically vibrated at $72 \mathrm{~Hz}$ by hand-held vibrator (Vyper 2.0, Max Recovery, São Paulo, SP) for 33sec (Figure 2). The vibrator was firmly held against a marked point in line with medial malleolus on the Achilles tendon by the same rater in all experimental sessions without causing any visible increments in resting plantar flexor torque. Ten seconds after vibration onset, five 2-s bursts of $20-\mathrm{Hz}$ tibial nerve NMES were applied with a 2-s rest between bursts. A 0.5 -s window starting $0.5,2$, and $4 \mathrm{~s}$ after NMES cessation was used to quantify Tvib, whilst Tsust was quantified $0.5,1,2$, and $3 \mathrm{~s}$ after vibration cessation. 


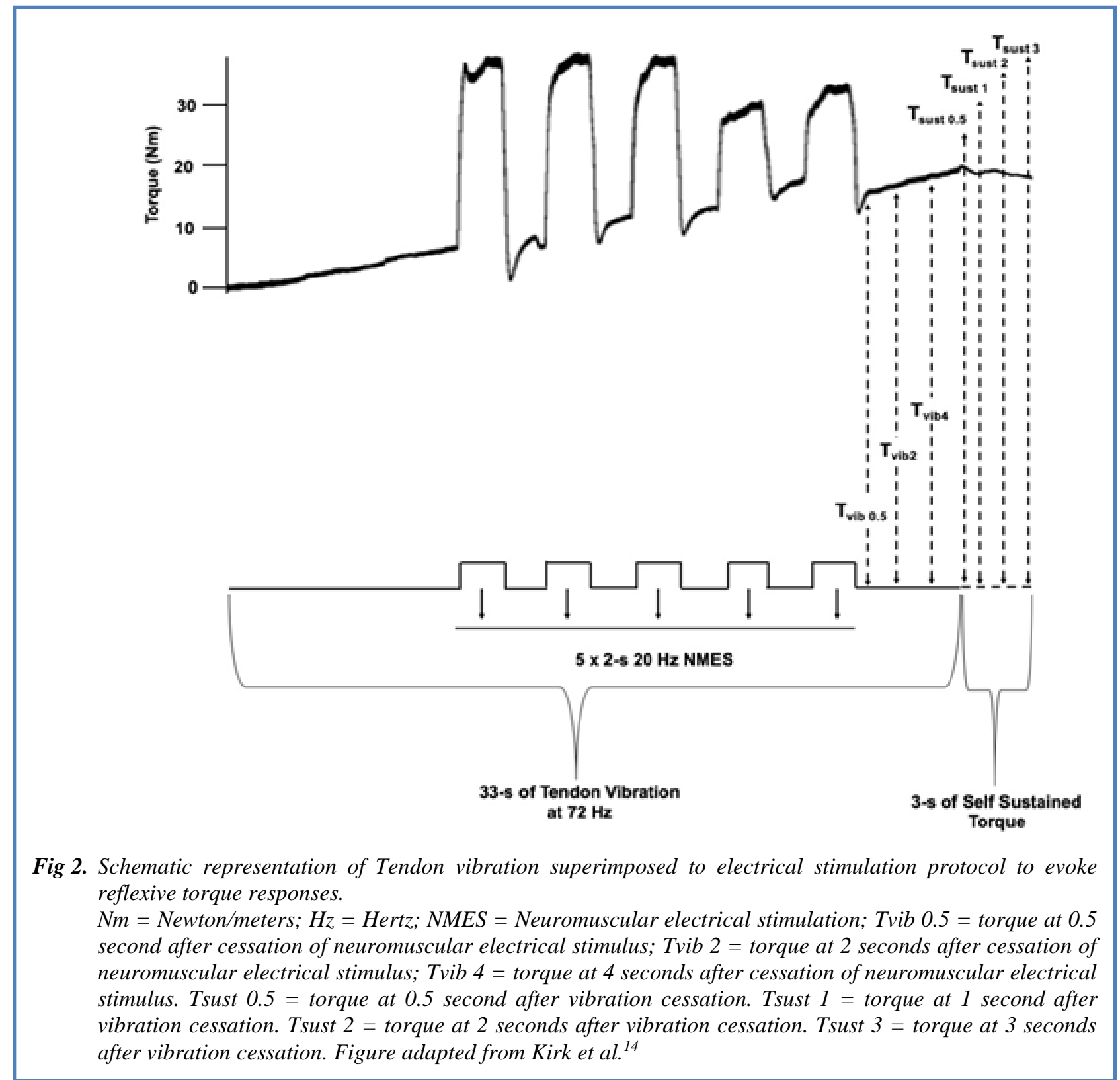

The warm-up effect was defined as the difference in torque developed during vibration $0.5 \mathrm{~s}$ after the first NMES burst (0.5-s window) to that at $0.5 \mathrm{~s}$ after the last NMES burst. Responsiveness to the vib+stim protocol was considered as a visible warm-up effect or notable sustained torque following the vib+stim sequence 14 . As plantar flexor muscles impose a small passive torque even when the muscle is relaxed, ${ }^{14,19,20}$ the baseline torque was subtracted before Tvib and Tsust calculation. Ankle joint torque and the electrical stimulus were simultaneously recorded using LabChart software (version 6.1.3, PowerLab system ADInstruments, NSW, Australia).

\section{Statistical analysis}

Data distribution was verified by Shapiro-wilk test. The mean and standard deviations (SD) for Tvib and Tsust scores were calculated to quantify inter- and intrasession reliabilities. Relative reliability was assessed by two-way mixed effect intraclass correlation coefficient (ICC type 3.1) with absolute agreement. ${ }^{21,22}$ We denoted ICC scores $<0.5$ as poor, 0.5 to 0.74 as moderate, 0.75 to 0.9 as good, and $>0.9$ as excellent. ${ }^{22}$ Typical error (TE) and withinparticipant coefficient of variation (CV) were used to assess absolute reliability. ${ }^{21,23,24} \mathrm{TE}$ was calculated as SD of the difference divided by square root of $2,{ }^{24}$ while CV was calculated for each participant dividing the SD of each pair of measurements by its mean multiplied by $100 .^{23}$ Bland and Altman analysis and paired t-tests or Wilcoxon tests (non-normality data, i.e., Tsust at 2 and 3 $\mathrm{s}$ after NMES and vibration cessation) were used to assess systematic error. ${ }^{23}$ The worthwhile changes in Tvib and Tsust were quantified as small (SWC), moderate (MWC), and large (LWC) according to the 
Table 1. Intra-session reliability scores of vibratory and sustained torque ( $\mathrm{Nm})$.

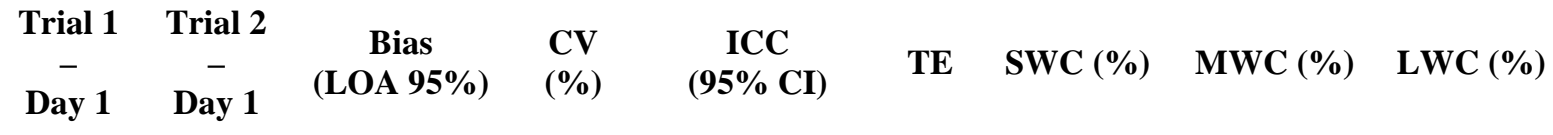

\begin{tabular}{|c|c|c|c|c|c|c|c|c|c|}
\hline $\begin{array}{c}\mathbf{T}_{\text {vib- }} \\
0.5 \mathrm{~s}\end{array}$ & $\begin{array}{c}8.3 \pm \\
7.2\end{array}$ & $\begin{array}{c}9.2 \pm \\
5.7\end{array}$ & $\begin{array}{c}-0.9 \\
(-12 \text { to } 10)\end{array}$ & 51.1 & $\begin{array}{c}0.76 \\
(0.23-0.93)\end{array}$ & 4.0 & $\begin{array}{c}1.2 \\
(13.4 \%)\end{array}$ & $\begin{array}{c}3.5 \\
(40.1 \%)\end{array}$ & $\begin{array}{c}7.0 \\
(80.3 \%)\end{array}$ \\
\hline $\mathbf{T}_{\text {vib-2s }}$ & $\begin{array}{c}9.1 \pm \\
7.0\end{array}$ & $\begin{array}{c}10.7 \pm \\
5.1\end{array}$ & $\begin{array}{c}-1.6 \\
(-13 \text { to } 9.3)\end{array}$ & 40.9 & $\begin{array}{c}0.74 \\
(0.14-0.92)\end{array}$ & 4.0 & $\begin{array}{c}1.1 \\
(11 \%)\end{array}$ & $\begin{array}{c}3.3 \\
(33.1 \%)\end{array}$ & $\begin{array}{c}6.5 \\
(66.1 \%)\end{array}$ \\
\hline $\mathbf{T}_{\text {vib-4s }}$ & $\begin{array}{c}9.8 \pm \\
6.9\end{array}$ & $\begin{array}{c}11.4 \pm \\
5.2\end{array}$ & $\begin{array}{c}-1.6 \\
(-12 \text { to } 8.5)\end{array}$ & 37.0 & $\begin{array}{c}0.79 \\
(0.30-0.94)\end{array}$ & 3.6 & $\begin{array}{c}1.1 \\
(10.5 \%)\end{array}$ & $\begin{array}{c}3.4 \\
(31.5 \%)\end{array}$ & $\begin{array}{c}6.7 \\
(63 \%)\end{array}$ \\
\hline $\begin{array}{c}\mathbf{T}_{\text {sust- }} \\
0.5 \mathrm{~s}\end{array}$ & $\begin{array}{c}10.2 \pm \\
6.8\end{array}$ & $\begin{array}{c}10.5 \pm \\
5.6\end{array}$ & $\begin{array}{c}-0.3 \\
(-12 \text { to } 11)\end{array}$ & 35.3 & $\begin{array}{c}0.73 \\
(0.12-0.92)\end{array}$ & 4.1 & $\begin{array}{c}1.1 \\
(10.7 \%)\end{array}$ & $\begin{array}{c}3.3 \\
(32.2 \%)\end{array}$ & $\begin{array}{c}6.7 \\
(64.4 \%)\end{array}$ \\
\hline $\begin{array}{c}\mathbf{T}_{\text {sust- }} \\
\text { 1s }\end{array}$ & $\begin{array}{c}8.1 \pm \\
5.4\end{array}$ & $\begin{array}{c}8.0 \pm \\
6.0\end{array}$ & $\begin{array}{c}0.1 \\
(-9.1 \text { to } 9.4)\end{array}$ & 38.5 & $\begin{array}{c}0.80 \\
(0.33-0.94)\end{array}$ & 3.3 & $\begin{array}{c}1.0 \\
(13 \%)\end{array}$ & $\begin{array}{c}3.1 \\
(39 \%)\end{array}$ & $\begin{array}{c}6.2 \\
(78 \%)\end{array}$ \\
\hline $\begin{array}{c}\mathbf{T}_{\text {sust- }} \\
\text { 2s }\end{array}$ & $\begin{array}{c}4.7 \pm \\
5.0\end{array}$ & $\begin{array}{c}5.3 \pm \\
5.9\end{array}$ & $\begin{array}{c}-0.7 \\
(-7.5 \text { to } 6.2)\end{array}$ & 53.6 & $\begin{array}{c}0.89 \\
(0.63-0.97)\end{array}$ & 2.5 & $\begin{array}{c}1.0 \\
(20.9 \%)\end{array}$ & $\begin{array}{c}3.1 \\
(62.6 \%)\end{array}$ & $6.2(125 \%)$ \\
\hline $\begin{array}{c}\mathbf{T}_{\text {sust- }} \\
\text { 3s }\end{array}$ & $\begin{array}{c}3.8 \pm \\
4.3\end{array}$ & $\begin{array}{c}4.7 \pm \\
5.8\end{array}$ & $\begin{array}{c}-0.9 \\
(-8.3 \text { to } 6.5)\end{array}$ & 71.3 & $\begin{array}{c}0.84 \\
(0.48-0.95)\end{array}$ & 2.7 & $\begin{array}{c}1.0 \\
(22.6 \%)\end{array}$ & $\begin{array}{c}2.9 \\
(67.8 \%)\end{array}$ & 5.7 (136\%) \\
\hline
\end{tabular}

Tvib = vibratory torque; Tsust = sustained torque; $L O A=$ Limit of agreement $; C V=$ within-participant coefficient of variation; ICC = Intraclass correlation coefficient; TE = Typical error of the measurement; SWC = Smallest worthwhile change; $M W C=$ Moderate worthwhile change; $L W C=$ Large worthwile change.

following formula: 0.2 (small), 0.6 (moderate), and 1.2 (large) X between-participant SD. ${ }^{25,26}$ Statistical significance was set to $\mathrm{p}<0.05$. The Shapiro-wilk test, ICC, paired t-test, and Wilcoxon procedures were completed in the Statistical Package for Social Sciences (SPSS, version 20.0). SWC, MWC, LWC, TE and CV were performed in a custom-made Microsoft Excel spreadsheet. Bland and Altman analysis were completed in GraphPad Prism (Version 8).

\section{Results}

Thirteen of the fifteen participants demonstrated a warmup effect or a notable self-sustained torque after vib+stim, i.e., $87 \%$ of the sample showed Tvib and Tsust responses to the vib+stim protocol using NMES over the nerve. The intrasession reliability scores varied according to the time point at which the measurements were taken during vib+stim protocol, and are reported in Table 1. The highest intrasession ICCs for Tvib and Tsust were 0.79 and 0.89 , respectively. The lowest intra- session TEs and CVs were $3.6 \mathrm{Nm}$ and $37 \%$ for Tvib, and $2.5 \mathrm{Nm}$ and $35 \%$ for Tsust. No significant systematic intra-session bias was found $(p>0.05)$ with the lowest intra-session bias being $-0.9 \mathrm{Nm}$ for Tvib and $0.1 \mathrm{Nm}$ for Tsust. The lowest SWC, MWC, and LWC were 10.5\%, $31.5 \%$, and $63 \%$, respectively, for Tvib, and $10.7 \%$, $32.2 \%$ and $64.4 \%$, respectively, for Tsust.

The inter-session reliability of Tvib and Tsust also varied according to the time-point at which the measurements were taken during and after the vib+stim protocol, as reported in Table 2. The highest inter-session ICCs for Tvib and Tsust were 0.43 and 0.56 , respectively. The lowest inter-session TEs and CVs were 5.4 Nm and 53\% for Tvib, and $4.5 \mathrm{Nm}$ and $49 \%$ for Tsust. No significant systematic inter-session bias was found $(p>0.05)$ and the lowest inter-session biases were $0.9 \mathrm{Nm}$ for Tvib and 0.1 Nm for Tsust. The lowest SWC, MWC, and LWC were $14.1 \%, 42.2 \%$ and $84.4 \%$, respectively, for Tvib, and $13.7 \%, 41.1 \%$ and $82.3 \%$, respectively, for Tsust. 
Table 2. Inter-session reliability scores of vibratory torque and sustained torque at different time-points (Nm).

$\begin{array}{ccccccccc}\text { Trial 1 } & \text { Trial 1 } & \text { Bias } & \text { CV } & \text { ICC } & \text { TE } & \text { SWC } & \text { MWC } & \text { LWC } \\ - & - & \text { (\%) } & \text { (\%) } & (\%)\end{array}$

\begin{tabular}{|c|c|c|c|c|c|c|c|c|c|}
\hline $\begin{array}{c}\mathbf{T}_{\text {vib- }} \\
0.5 \mathrm{~s}\end{array}$ & $8.3 \pm 7.2$ & $\begin{array}{c}6.9 \pm \\
5.2\end{array}$ & $\begin{array}{c}1.4 \\
(-15 \text { to } 18)\end{array}$ & 75.4 & $\begin{array}{c}0.16 \\
(-1.76-0.74)\end{array}$ & 6.0 & $\begin{array}{c}1.3 \\
(17.3 \%)\end{array}$ & $\begin{array}{c}3.9 \\
(51.8 \%)\end{array}$ & $\begin{array}{c}7.8 \\
(103 \%)\end{array}$ \\
\hline $\mathbf{T}_{\mathbf{v i b}-2 \mathrm{~s}}$ & $9.1 \pm 7.0$ & $\begin{array}{c}8.1 \pm \\
5.7\end{array}$ & $\begin{array}{c}1.0 \\
(-15 \text { to } 17)\end{array}$ & 63.1 & $\begin{array}{c}0.3 \\
(-1.30-0.79)\end{array}$ & 5.8 & $\begin{array}{c}1.3 \\
(15.7 \%)\end{array}$ & $\begin{array}{c}4.0 \\
(47 \%)\end{array}$ & $\begin{array}{c}8.1 \\
(93.9 \%)\end{array}$ \\
\hline $\mathbf{T}_{\mathbf{v i b}-4 \mathrm{~s}}$ & $9.8 \pm 6.9$ & $\begin{array}{c}8.9 \pm \\
5.6\end{array}$ & $\begin{array}{c}0.9 \\
(-14 \text { to } 16)\end{array}$ & 53.1 & $\begin{array}{c}0.43 \\
(-0.89-0.83)\end{array}$ & 5.4 & $\begin{array}{c}1.3 \\
(14.1 \%)\end{array}$ & $\begin{array}{c}4.0 \\
(42.2 \%)\end{array}$ & $\begin{array}{c}7.9 \\
(84.4 \%)\end{array}$ \\
\hline $\begin{array}{c}\mathbf{T}_{\text {sust- }} \\
0.5 \mathrm{~s}\end{array}$ & $\begin{array}{c}10.2 \pm \\
6.8\end{array}$ & $\begin{array}{c}8.9 \pm \\
5.3\end{array}$ & $\begin{array}{c}1.3 \\
(-12 \text { to } 13)\end{array}$ & 49.3 & $\begin{array}{c}0.56 \\
(-0.45-0.87)\end{array}$ & 4.8 & $\begin{array}{c}1.3 \\
(13.7 \%)\end{array}$ & $\begin{array}{c}3.9 \\
(41.1 \%)\end{array}$ & $\begin{array}{c}7.8 \\
(82.3 \%)\end{array}$ \\
\hline $\begin{array}{c}\mathbf{T}_{\text {sust- }} \\
\text { 1s }\end{array}$ & $8.1 \pm 5.4$ & $\begin{array}{c}6.7 \pm \\
5.4\end{array}$ & $\begin{array}{c}1.4 \\
(-12 \text { to } 15)\end{array}$ & 51.5 & $\begin{array}{c}0.37 \\
(-1.05-0.81)\end{array}$ & 4.8 & $\begin{array}{c}1.2 \\
(16.5 \%)\end{array}$ & $\begin{array}{c}3.6 \\
(49.4 \%)\end{array}$ & $\begin{array}{c}7.3 \\
(98.8 \%)\end{array}$ \\
\hline $\begin{array}{c}\mathbf{T}_{\text {sust- }} \\
2 \mathrm{~s}\end{array}$ & $4.7 \pm 5.0$ & $\begin{array}{c}4.5 \pm \\
5.5\end{array}$ & $\begin{array}{c}0.2 \\
(-14 \text { to } 14)\end{array}$ & 91.0 & $\begin{array}{c}0.10 \\
(-1.95-0.73)\end{array}$ & 5.1 & $\begin{array}{c}1.2 \\
(25.9 \%)\end{array}$ & $\begin{array}{c}3.5 \\
(77.8 \%)\end{array}$ & $\begin{array}{c}7.1 \\
(156 \%)\end{array}$ \\
\hline $\begin{array}{c}\mathbf{T}_{\text {sust- }} \\
\text { 3s }\end{array}$ & $3.8 \pm 4.3$ & $\begin{array}{c}3.7 \pm \\
5.3\end{array}$ & $\begin{array}{c}0.1 \\
(-12 \text { to } 12)\end{array}$ & 88.0 & $\begin{array}{c}0.26 \\
(-1.42-0.78)\end{array}$ & 4.5 & $\begin{array}{c}1.1 \\
(30.1 \%)\end{array}$ & $\begin{array}{c}3.4 \\
(90.3 \%)\end{array}$ & $\begin{array}{c}6.8 \\
(181 \%)\end{array}$ \\
\hline
\end{tabular}

Tvib = vibratory torque; Tsust = sustained torque $; L O A=$ Limit of agreement $; C V=$ within-participant coefficient of variation; ICC = Intraclass correlation coefficient; TE = Typical error of the measurement; SWC = Smallest worthwhile change; $M W C=$ Moderate worthwhile change; $L W C=$ Large worthwhile change.

\section{Discussion}

The present data show that Tsust and Tvib could be evoked in the plantar flexors by the vib+stim protocol using NMES over the nerve trunk in a young, healthy, male cohort. In addition, the intra-session Tsust and Tvib analyses revealed no significant systematic bias and moderate-to-good ICCs (>0.73). However, the confidence interval for ICCs was large and CVs were high (>10\%). Therefore, even within the same session, the test reliability may not be sufficient for research or clinical use. In fact, the degree of variation (error) of the measurement in vib+stim protocol was sufficiently high that, generally, only large changes in Tsust and Tvib would be confidently detected within the same session (i.e. LWC > TE > SWC and MWC). Inter-session Tvib and Tsust were also had poor reliability, indicating that the test may not be useful to track longitudinal changes in motoneuron facilitation capacity over time. Although, there was no significant systematic inter-session bias, Tvib and Tsust were associated with low ICCs and high
CVs at all measured time points. Furthermore, similar to intra-session results, only large changes in Tvib and Tsust would be detected across the sessions (i.e. $>63$ and $64 \%$ for Tvib and Tsust, respectively). The large intersession variability brings into question the utility of the vib+stim test, as conducted in the current experiments, for use in research and clinical settings. Previous studies have shown that Tsust and Tvib responses can be evoked by the vib+stim protocol with stimulation applied over the plantar flexor muscle belly. ${ }^{1,14}$ Kirk et al. ${ }^{14}$ reported that only $\sim 68 \%$ of the participants showed Tsust and Tvib responses using that method. In the present study, Tsust and Tvib responses were evoked using NMES over the nerve in 13 of the 15 participants (i.e. $87 \%$ of the sample). Although these findings are consistent with a greater $\mathrm{H}$ reflex responses being evoked by NMES applied over the trunk than the muscle belly, suggesting a potentiation of Ia afferent excitatory synapses onto spinal motoneurons and, possibly, favorable PIC development. ${ }^{2,16,27}$ Due to methodological and sample differences, the direct 


\section{Concurrent Achilles tendon vibration and tibial nerve stimulation}

Eur J Transl Myol 31 (4): 10045, 2021 doi: 10.4081/ejtm.2021.10045

comparison between our and Kirk et al. ${ }^{14}$ study should be considered cautiously and may not indicate adequately whether the nerve-targeted NMES is a more robust method for providing motoneuron facilitation than muscle belly NMES during concurrent Achilles tendon vibration and NMES protocol. Thus, future studies using similar methodological procedures and the same sample participants should be required. Nonetheless, reliability of the nerve-targeted technique was only poor to good, as demonstrated by high intra-session TEs and CVs (>35\%), and only moderate-to-good ICCs. Intra-session variability of the nerve stimulation method may be partly attributable to the NMES procedures. Indeed, repetitive NMES may elicit a progressive increase in PIC affecting motoneuronal excitability, ${ }^{1}$ which may persist for several minutes after cessation of the NMES. ${ }^{28}$ Therefore, given the effect of PICs on motoneuronal excitability, Tvib and Tsust might be expected to be higher in the second than the first trial when using the nerve stimulation method, which in turn was confirmed by our intra-session BlandAltman analysis. The poor to good reliability obtained using the nerve-targeted technique performed in the current study can be contrasted with the data of Trajano et al. ${ }^{1}$ who reported ICCs $>0.95$, indicating excellent intra-session reliability using muscle belly stimulation (although other indications of reliability were not provided). In addition to the different method of stimulation between the methods, discrepancies in intrasession reliability may result from other between-study differences such as subject heterogeneity, e.g. men and women participated in the study of Trajano et al. ${ }^{1}$ In addition to questionable intra-session reliability, Tsust and Tvib responses were also found not to be reliable between sessions. Whilst no other studies have reported inter-session reliability vib+stim outcomes, the present results are consistent with other electrophysiological techniques used to assess central activity, for which a large inter-session variability has been reported. ${ }^{29,30}$ Indeed, when assessed in a similar body position to that used in the current study (i.e. knee fully extended) medial gastrocnemius H-reflex inter-session reliability has also been shown to be poor. ${ }^{30}$ Although the reason for the poor inter-session reliability remains unclear, inconsistency in the measurement protocol may play a role. ${ }^{23}$ For example, whilst all trials were performed by the same experienced investigator, factors such as electrode positions and both hand-held vibrator position and pressure may have varied between sessions. The specific effects of these may be examined in future. Since PICs are an important neurophysiologic mechanism associated with multiple muscle outcomes including force production and spasticity, ${ }^{2,7,8}$ and it is well recognized that currently-accepted techniques for estimating PICs in humans are difficult to apply in many clinical populations, ${ }^{9,13}$ the development of other techniques is an important goal. The vib+stim shows promise in this regard, however the poor intra- and inter-session reliability of the data obtained when applying NMES over the nerve branch calls into question its use in research and clinical setting. ${ }^{23,24}$ This study has a potential limitation that should be mentioned, as the absence of muscle activity and motor unit recording, which in turn during nerve-target NMES makes difficult to know which muscles and motor units were really involved in vibratory and sustained torque as an estimate of PICs. However, the aim of vib+stim protocol is to be useful in a clinical setting without the use of a complex technology such as EMG system or complex algorithms, and torque production in the absence of synaptic input could indicate a self-sustained motor unit firing and has been used as a marker of PICs. ${ }^{1,14,31}$ In addition, inconsistency in the measurement protocol may play a role in the results. For example, whilst all trials were performed by the same experienced investigator, factors such as electrode positions and both hand-held vibrator position and pressure may have varied between sessions. The specific effects of these may be examined in future. The vib+stim protocol imposed by applying NMES over the tibial nerve tends to evoke Tvib and Tsust responses in a majority of individuals, and with likely a greater rate (i.e. higher proportion of 'responders') than when NMES is applied to the plantar flexor muscle belly. However, Tvib and Tsust responses showed unacceptable withinand between-day reliability, indicated by large withinparticipant variability, low ICC, and insensitivity to detect small-to-moderate worthwhile changes within and between sessions. Thus, at least when using the procedures adopted in the present study, the vib+stim technique using tibial nerve NMES may not be of use in the study of motoneuron facilitation, or PIC strength, in research or clinical environments.

\section{List of acronyms}

$$
\begin{aligned}
& \mathrm{Ca}^{2+}=\text { Calcium } \\
& \mathrm{CV}=\text { Coefficients of variation } \\
& \text { EMG = Electromyography } \\
& \mathrm{Hz}=\text { Hertz } \\
& \text { ICC = Intraclass correlation coefficients } \\
& \text { LWC = large worthwhile change } \\
& \text { MVC = Maximal voluntary contraction } \\
& \mathrm{MWC}=\text { moderate worthwhile change } \\
& \mathrm{Na}^{+}=\text {Sodium }
\end{aligned}
$$$$
\text { NMES = Neuromuscular electrical stimulation }
$$$$
\mathrm{p}=\text { alpha level }
$$$$
\text { PIC }=\text { Persistent inward currents }
$$$$
\mathrm{SD}=\text { Standard deviation }
$$$$
\text { SWC = small worthwhile change }
$$$$
\mathrm{TE}=\text { Typical Error }
$$$$
\text { Tsust }=\text { Sustained Torque }
$$$$
\text { Tvib = Vibratory Torque }
$$$$
\mathrm{Vib}+\text { stim }=\text { Concurrent Achilles tendon vibration and }
$$
neuromuscular electrical stimulation

\section{Authors' contributions}

DCLV participated in the conception and design of the work, data acquisition and analysis, drafting of the 


\section{Concurrent Achilles tendon vibration and tibial nerve stimulation}

Eur J Transl Myol 31 (4): 10045, 2021 doi: 10.4081/ejtm.2021.10045

manuscript, and approved the final version. AV participated in the conception and design of the work, data analysis, drafting of the manuscript, and approved the final version. MAS participated in the data acquisition, drafting of the manuscript, and approved final version. RRC participated in the data acquisition, drafting of the manuscript, and approved final version. VL participated in the data acquisition, drafting of the manuscript, and approved final version. AJB participated in the data analysis, drafting of the manuscript, revised of the English grammar, and approved the final version. MB participated in the conception and design of the work, data analysis, drafting of the manuscript, and approved the final version.

\section{Acknowledgments}

The authors would like to thank the volunteers for giving your time to participate of this experiment.

\section{Funding}

This research did not receive any specific grant from funding agencies in the public, commercial, or not-forprofit sectors.

\section{Conflict of Interest}

The authors declare no competing interests.

\section{Ethical Publication Statement}

We confirm that we have read the Journal's position on issues involved in ethical publication and affirm that this report is consistent with those guidelines.

\section{Corresponding Author}

Denis César Leite Vieira, Faculdade de Educação Física. University of Brasilia, Campus Universitário Darcy Ribeiro, Asa Norte, Brasília, DF, Brasil.

Zip code:70910-970. Phone: +55-61-31072522

ORCID iD: 0000-0002-0761-1846

E-mail: denisclvieira@hotmail.com

E-mails and ORCID iD of co-authors

Amilton Vieira: acmribeirao@gmail.com

ORCID iD: 0000-0002-6027-4367

Matheus Santos: matheusavelino1999@gmail.com

ORCID iD: 0000-0002-1795-0403

Rafael Cunha: rafaelcunha05@gmail.com

ORCID iD: 0000-0002-1865-2790

Victor Lage: victorlage@gmail.com

ORCID iD: 0000-0001-6322-9804

Anthony Blazevich: a.blazevich@ecu.edu.au

ORCID iD: 0000-0003-1664-1614

Martim Bottaro: martim@unb.br

ORCID iD: 0000-0002-4315-3176

\section{References}

1. Trajano GS, Seitz LB, Nosaka K, Blazevich AJ. Can passive stretch inhibit motoneuron facilitation in the human plantar flexors? J Appl Physiol.
2014;117(12):1486-1492. doi:10.1152/japplphysiol .00809 .2014

2. Heckman CJ, Gorassini MA, Bennett DJ. Persistent inward currents in motoneuron dendrites: Implications for motor output. Muscle Nerve. 2005;31(2):135-156. doi:10.1002/mus.20261.

3. Lee RH, Heckman CJ. Paradoxical Effect of QX314 on Persistent Inward Currents and Bistable Behavior in Spinal Motoneurons In Vivo. J Neurophysiol. 1999;82(5):2518-2527. doi:10.1152 /jn.1999 . 82.5.2518.

4. Gorassini MA, Knash ME, Harvey PJ, Bennett DJ, Yang JF. Role of motoneurons in the generation of muscle spasms after spinal cord injury. Brain. 2004;127(10):2247-2258. doi:10.1093/brain/awh 243.

5. Kirk EA, Rice CL. Contractile function and motor unit firing rates of the human hamstrings. J Neurophysiol. 2017;117(1):243-250. doi:10.1152 /jn.00620.2016.

6. Del Vecchio A, Negro F, Holobar A, Casolo A, Folland JP, Felici F, Farina D. You are as fast as your motor neurons: speed of recruitment and maximal discharge of motor neurons determine the maximal rate of force development in humans. J Physiol. 2019 May;597(9):2445-2456. doi: 10.1113/JP277396. Epub 2019 Mar 1.

7. Johnson MD, Heckman CJ. Interactions between focused synaptic inputs and diffuse neuromodulation in the spinal cord. Ann N Y Acad Sci. 2010;1198(1):35-41. doi:10.1111/j.1749-6632 .2010.05430.x.

8. Heckman CJ, Johnson M, Mottram C, Schuster J. Persistent inward currents in spinal motoneurons and their influence on human motoneuron firing patterns. Neuroscientist. 2008;14(3):264-275. doi:10.1177/1073858408314986.

9. Vandenberk MS, Kalmar JM. An evaluation of paired motor unit estimates of persistent inward current in human motoneurons. J Neurophysiol. 2014;111(9):1877-1884. doi:10.1152/jn.00469.2013.

10. Bennett DJ, Li Y, Harvey PJ, Gorassini M. Evidence for Plateau Potentials in Tail Motoneurons of Awake Chronic Spinal Rats With Spasticity. J Neurophysiol. 2001;86(September 2000):1972-1982. doi: 10.1152/jn.2001.86.4.1972.

11. Perrier J-F, Rasmussen HB, Christensen RK, Petersen AV. Modulation of the intrinsic properties of motoneurons by serotonin. Curr Pharm Des. 2013;19(stage 28):4371-4384. doi:10.2174/138161 28113199990341.

12. Hyngstrom AS, Johnson MD, Heckman CJ. Summation of excitatory and inhibitory synaptic inputs by motoneurons with highly active dendrites. J Neurophysiol. 2008;99(4):1643-1652. doi:10. 1152/jn.01253.2007.

13. Mottram CJ, Suresh NL, Heckman CJ, Gorassini MA, Rymer WZ. Origins of abnormal excitability in biceps brachii motoneurons of spastic-paretic 


\section{Concurrent Achilles tendon vibration and tibial nerve stimulation}

Eur J Transl Myol 31 (4): 10045, 2021 doi: 10.4081/ejtm.2021.10045

stroke survivors. J Neurophysiol. 2009;102(4): 2026-2038. doi:10.1152/jn.00151.2009.

14. Kirk BJC, Trajano GS, Pulverenti TS, Rowe G, Blazevich AJ. Neuromuscular Factors Contributing to Reductions in Muscle Force After Repeated, High-Intensity Muscular Efforts. Front Physiol. 2019;10(June):1-17. doi:10.3389/fphys.2019.00783.

15. Magalhães FH, Kohn AF. Vibration-induced extra torque during electrically- evoked contractions of the human calf muscles. J Neuroeng Rehabil. 2010;7(26):1-17. doi:10.1186/1743-0003-7-26.

16. Bergquist AJ, Clair JM, Collins DF. Motor unit recruitment when neuromuscular electrical stimulation is applied over a nerve trunk compared with a muscle belly: Triceps surae. J Appl Physiol. 2011;110(3):627-637. doi:10.1152/japplphysiol. 01103.2010.

17. Bergquist AJ, Wiest MJ, Collins DF. Motor unit recruitment when neuromuscular electrical stimulation is applied over a nerve trunk compared with a muscle belly: Quadriceps femoris. J Appl Physiol. 2012;113(1):78-89. doi:10.1152/jappl physiol.00074.2011.

18. Lee RH, Heckman CJ. Bistability in spinal motoneurons in vivo: Systematic variations in rhythmic firing patterns. J Neurophysiol. 1998; 80(2):572-582. doi:10.1152/jn.1998.80.2.572.

19. Mizuno T. Changes in joint range of motion and muscle-tendon unit stiffness after varying amounts of dynamic stretching. J Sports Sci. 2017; 35(21):2157-2163. doi:10.1080/02640414.2016. 1260149.

20. Opplert J, Babault N. Acute effects of dynamic stretching on mechanical properties result from both muscle-tendon stretching and muscle warmup. J Sport Sci Med. 2019;18(2):351-358.

21. Weir JP. Quantifying Test-Retest Reliability Using the Intraclass Correlation Coefficient and the SEM. J Strength Cond Res. 2005;19(1):231. doi:10. 1519/15184.1.

22. Koo TK, Li MY. A Guideline of Selecting and Reporting Intraclass Correlation Coefficients for
Reliability Research. J Chiropr Med. 2016;15(2):155-163. doi:10.1016/j.jcm.2016.02. 012.

23. Atkinson G, Nevill AM. Statistical Methods For Assessing Measurement Error (Reliability) in Variables Relevant to Sports Medicine. Sport Med. 1998;26(4):217-238. doi:10.2165/00007256-1998 26040-00002.

24. Hopkins WG. Measures of Reliability in Sports Medicine and Science. Sport Med. 2000;30(1):115. doi:10.2165/00007256-200030010-00001.

25. Hopkins WG. How to Interpret Changes in an Athletic Performance Test. Sportscience. 2004;8:17.

26. Flanagan EP. The effect size statistic-applications for the strength and conditioning coach. Strength Cond J. 2013;35(5):37-40. doi:10.1519/SSC.0b013 e3182a64d20.

27. Collins DF. Central Contributions to Contractions Evoked by Tetanic Neuromuscular Electrical Stimulation. Exerc Sport Sci Rev. 2007;103(1):102109. doi:10.1097/jes.0b013e3180a0321b.

28. Kitago T, Mazzocchio R, Liuzzi G, Cohen LG. Modulation of H-reflex excitability by tetanic stimulation. Clin Neurophysiol. 2004;115(4):858861. doi:10.1016/j.clinph.2003.11.029.

29. Leung H, Latella C, Lamon S, Hendy AM. The reliability of neurological measurement in the vastus medialis: Implications for research and practice. Front Psychol. 2018;9(OCT):1-10. doi:10.3389/fpsyg.2018.01857.

30. Merlet AN, Cattagni T, Cornu C, Jubeau M. Effect of knee angle on neuromuscular assessment of plantar flexor muscles: A reliability study. PLoS One. 2018;13(3):1-16. doi:10.1371/journal.pone. 0195220.

31. Trajano GS, Taylor JL, Orssatto LBR, McNulty CR, Blazevich AJ. Passive muscle stretching reduces estimates of persistent inward current strength in soleus motor units. J Exp Biol. 2020;223(September). doi:10.1242/jeb.229922.

Submission: August 16, 2021

Revision submitted: October 20, 2021 Accepted for publication: October 22, 2021 